\title{
Adapting Publish/Subscribe Middleware to Achieve Gnutella-like Functionality
}

\author{
Dennis Heimbigner \\ Department of Computer Science \\ University of Colorado, Boulder \\ dennis@cs.colorado.edu
}

\section{Keywords}

Middleware, peer-to-peer, publish-subscribe, Gnutella.

\begin{abstract}
Gnutella represents a new wave of peer-to-peer applications providing distributed discovery and coordinated sharing of resources across the Internet. Gnutella is distinguished by its support for anonymity and by its decentralized architecture. The current Gnutella architecture and protocol have numerous flaws with respect to efficiency, anonymity, and vulnerability to malicious actions. An alternative design is described providing Gnutella-like functionality but removing or mitigating many of Gnutella's flaws. This design, referred to as Query/Advertise (Q/A) is based upon a scalable Publish/Subscribe middleware system called Siena. A prototype implementation of $\mathrm{Q} / \mathrm{A}$ is described. The relative benefits of this approach are discussed, and a number of open research problems are identified with respect to $\mathrm{Q} / \mathrm{A}$ systems.
\end{abstract}

\section{INTRODUCTION}

Gnutella [9] represents a new wave of peer-to-peer [17][20] applications providing distributed discovery and sharing of resources in wide-area networks such as the Internet. Gnutella is distinguished by its support for anonymity and by its decentralized architecture. This is in contrast to Napster [19], for example, which is similar, but is more centralized. The Gnutella protocol provides a simple request-response paradigm for sharing files directly between peer computers. Users send out requests for files, and these requests are propagated to all peer nodes in the Gnutella net. In response, nodes generate replies back to the query originator indicating that they have the specified file or files.

Gnutella is biased towards the sharing of files, but it and similar applications are being used for managing other kinds of resources. Intel [15] for example, is using the technology to solve chip design problems by finding and using spare cycles on the company's machines. A Peer-to-Peer Working Group [20] has been formed to standardize and exploit this kind of application,

Permission to make digital or hard copies of all or part of this work for personal or classroom use is granted without fee provided that copies are not made or distributed for profit or commercial advantage and that copies bear this notice and the full citation on the first page. To copy otherwise, or republish, to post on servers or to redistribute to lists, requires prior specific permission and/or a fee.

SAC 2001, Las Vegas, NV.

Copyright 2000 ACM 1-58113-324-3/01/02 ..\$5.00. with Hewlett-Packard, IBM, and Intel as founding members.

The promise of Gnutella is marred by a large number of flaws in its protocol and architecture. In particular, the following problems have been identified [10][11].

- Imperfect anonymity. In practice, the anonymity of Gnutella is easy to compromise because peer node IP addresses are revealed at various points in its operation.

- Malicious users. Since every node potentially routes messages from other nodes, it is easy for malicious users to seriously affect the core operation of the Gnutella net.

- Efficiency. Gnutella has a well-deserved reputation for generating inordinate amounts of network traffic. In addition, slow nodes can create serious performance bottlenecks in the net.

- Query expressions. The format of Gnutella queries is not standardized. A query contains an arbitrary character string whose interpretation is entirely determined by any node that receives it.

The goal of this paper is to demonstrate that these flaws in Gnutella can be alleviated using a scalable Publish/Subscribe [4] middleware system adapted for distributed routing of messages across a wide-area network such as the Internet. The Publish/Subscribe paradigm is implemented by such systems as Siena [3], Tibco [23], and Elvin [22].

The Publish/Subscribe paradigm is normally used for asynchronous coordination of distributed systems. Publishers notify subscribers of interesting events. The subscribers may in turn perform actions in response to those events. This paper demonstrates that Publish/Subscribe can be used in quite a different fashion: as a basis for what will be termed the Query/Advertise (Q/A) paradigm.

In a Publish/Subscribe system, clients publish event (or notification) messages with highly structured content, and other clients make available a filter (a kind of pattern) specifying the subscription: the content of events to be received at that client. Event message distribution is handled by an underlying contentbased routing [6] network, which is a set of server nodes interconnected as a peer-to-peer network. The content-based router is responsible for sending copies of event messages to all clients whose filters match that message.

Analogous to Publish/Subscribe, the Query/Advertise (Q/A) paradigm is defined to represent Gnutella-like systems. Queries are represented by the contents of messages describing attributes of resources (e.g., files) of interest. Providers of resources 
establish advertisements describing the attributes of their available resources. Upon receiving a query, providers generate response messages whose content describes the detailed attributes of their specific resources. These responses are injected into the network and routed back to the query originator. The provision for responses represents a major difference between Q/A and Publish/Subscribe.

This paper is organized as follows. First, the architecture, operation, and problems of Gnutella are described in more detail. Then the architecture and operation of a specific Publish/Subscribe system is described. Next, it is shown how to implement Query/Advertise on a such a system and how it solves many of Gnutella's problems. A prototype implementation is also described. After that, research issues for Q/A are discussed, and finally there are some comparisons to related systems.

\section{GNUTELLA ARCHITECTURE AND PROTOCOL}

The Gnutella architecture consists of a dynamically changing set of nodes connected using TCP/IP. Each node acts as a client (an originator of queries), a server (a provider of file information, and as a router (a transmitter of queries and responses). The generic term node ${ }^{l}$ will be used to refer to this combination of functionality. At any given point in time, a Gnutella net consists of a set of interconnected nodes. A new Gnutella user starts an instance of the Gnutella node software. That node uses out-ofband means to locate another node and establish a connection to it. This extends the net and makes the new node's files available to all other nodes in the net.

Once connections are established, nodes use the Gnutella protocol to communicate. There is an initialization conversation following which nodes send out typed packets into the Gnutella net to locate and retrieve files. There are five kinds of packets.

1. QUERY - request to locate a set of files matching some filter criteria.

2. HITS - response to a query giving a list of files matching the filter criteria and the IP address of the provider; note that there may multiple responders to a given query.

3. PING - request for the transitive closure of connected nodes to identify themselves.

4. PONG - response by a node upon receiving a PING; the responding node provides its IP address and number of sharable files it contains.

5. PUSH - request for a file provider to contact the requester. This provides a simple mechanism to attempt to get through firewalls.

The PING, PONG, and PUSH packets are not considered further as they are not essential to the core operation of Gnutella.

The query-response cycle for a Gnutella node involves three steps. In the first step, a QUERY packet is sent out. The packet contains a string specifying the set of files of interest. Each node that receives the packet uses this string to determine which files, if any, match the query. Unfortunately, Gnutella defines no standard format or matching semantics for this string; its interpretation is

\footnotetext{
${ }^{1}$ Some developers of Gnutella use the term Servent.
}

completely determined by each node that receives it. In practice, the query string is interpreted as a literal substring or as a regular expression that is to be matched against local file name paths.

In the second step, a node that matches a query generates one or more HITS packets giving information about obtaining specific files. Again, there is no standard format, except that it is a list of null-terminated strings. As a rule, these strings specify URLs for each file, but because URLs contain IP addresses, the HITS packet breaks the anonymity of nodes.

In the third step, the query node connects directly to the response node and uses a simplified version of the HTTP [8] protocol to retrieve the file using the returned URL. Thus, it bypasses the Gnutella net altogether.

Message passing in Gnutella represents a form of spreading activation. That is, whenever a node receives a message, it sends copies out to all of its other connections. Obviously this can generate large amounts of redundant traffic. Gnutella relies on two mechanisms to reduce traffic. First, each message has a time-tolive (TTL) counter that is decremented on every transmission. Secondly, Nodes are expected to cache information about messages they receive and if they receive a duplicate, then they do not forward it. In spite of these features, Gnutella still generates a large number of messages. The protocol expects that response messages, such as PONG and HITS will not be widely disseminated, but will instead by returned along a specific path back to the generator of the original PING or QUERY message. This requires, of course, that intermediate sites track path information so that this kind of directed reply can be implemented. It has been observed that many available Gnutella software packages do not keep such information, thus causing reply messages to be widely propagated, which again increases message traffic unnecessarily.

\section{A PUBLISH/SUBSCRIBE ARCHITECTURE}

The Siena [3] Publish/Subscribe middleware system developed at the University of Colorado, will be used to provide a canonical architecture. Other similar systems exist and are discussed in Section 8 . We provide a somewhat detailed description of Siena in order to present the features necessary for its use in Query/Advertise.

Siena messages are structured as attribute-value pairs where attributes can have one of many possible types, such as string, date, integer, or double. An example message could be represented as the following set of tuples.

\{ (author, "Chandler") (title, "Playback") (firstedition, True) \}

A client establishes a subscription by specifying a filter pattern that specifies the kinds of messages it wishes to receive. A filter is a set of triples of (attribute, operator, value), where the operator is a comparison operator such as the usual arithmetic comparisons for numbers, or substring for strings. In order for a message to match a filter, every attribute in the message must satisfy all corresponding filter triples when the message value is substituted and the operator applied. Thus, all of the tuples may be considered to be logically ANDed together. A logical $O R$ can be achieved by specifying multiple separate filters. An example filter might be represented by the following set of tuples.

$\{$ (author, =, “Chandler") (firstedition, =, True) (price, $>, 400)\}$ 
It is important to note that the attribute names used in events and subscriptions have no inherent semantic meaning. As with all such attribute-based systems, there must be some externally defined and mutually agreed upon meaning for the attributes.

Siena adopts a peer-to-peer architecture where arbitrary Siena servers connect to form a specific topology. In the simplest case, a client connects to a server and establishes a subscription. The server then forwards the subscription filter to all of its peers. Each peer notes where the subscription came from, and forwards it to its peers. Later, when some other client connects to a server and generates an event message, the local copy of the filter can be applied at that server to determine the next server to which the message should be forwarded. Note that if a message is generated for which no filter matches at the local server, then it will not be forwarded at all and so will generate no inter-server traffic. This kind of content-based routing is analogous to IP routing in the Internet, but instead of specific IP addresses, the content of messages of determines the destination (or destinations) for the message - an important distinction with respect to anonymity.

Siena is specifically designed to scale well to wide-area networks. One way this is achieved is by providing an important optimization that can reduce the number of filters that a given server must maintain. Key to this optimization is the Covers relation over filters. At a given server, for any two filters, F1 and $\mathrm{F} 2$, say, it can be determined if F1 Covers F2 or F2 Covers F1, or neither. F1 Covers F2 if any message that matches F2 also matches F1; F1 is more general than F2. Using this relationship, a forest of partial order trees can be constructed over all filters. Siena servers need only propagate the filters that are at the root of each Covers ordering.

\section{QUERY/ADVERTISE USING PUBLISH/SUBSCRIBE}

A query/advertise system has three primary concepts: advertisements, queries, and responses. Using a Publish/Subscribe messaging system as a substrate on which to implement Query/Advertise (Q/A) involves mapping these three concepts to the concepts of the underlying Publish/Subscribe system.

1. Advertisements. Advertisements map to subscriptions. A client acting as a resource provider describes his available resources using a filter pattern and establishes a subscription based on that filter. In effect, the notion of a subscription is generalized to become a kind of content-based address where messages are sent to addresses based on their content. For Q/A, this address represents an advertisement describing queries for which it may be able to provide a response.

2. Queries. Queries map to event messages. A client constructs a message describing the resource in which it is interested, and then inserts the message into the Q/A system where it is distributed to all clients with matching advertisements. As with advertisements, the notion of event is being generalized to a message and is not tied specifically to event semantics. Note that the query must contain some kind of identification, a "return address" if you will, so that responses can be returned to the query originator.

3. Responses. Responses also map to (event) messages in the underlying Publish/Subscribe system. Each advertiser that receives a message must perform a detailed examination of its stock of resources and construct a message describing each available matching resource in more detail. This response is then routed back to the query originator. The response must include some identification for the matching advertiser so that the query originator can obtain the actual resource. For both query return addresses and advertiser addresses, note that the identity is generally not an IP address, but rather some arbitrary but unique set of attributes sufficient for routing, but capable of maintaining anonymity.

The above discussion glosses over some mismatches between a Publish/Subscribe system and a Q/A system. That discussion is deferred to Section 7.

\section{A PROTOTYPE IMPLEMENTATION}

A prototype Q/A system called Quad was developed using the existing publicly available Java-based Siena prototype [2]. As a result, much of the underlying Siena architecture and design shows through in Quad. This prototype supports query, advertise, and response, but does not support actually copying a resource such as a file from a responder to a query originator. See the separate technical report [12] for details.

Key to the operation of the prototype is its use of return addresses that mark queries with a "return address" (an arbitrary name) of the query originator. This return address is implicitly copied into the query, thus allowing the $\mathrm{Q} / \mathrm{A}$ servers to properly and efficiently route replies back to the query originator. Similarly, an address for the advertiser is also copied into any reply, which allows the query originator to communicate with the specific provider to obtain access to the resource. Of course, the provider could mimic Gnutella and provide a direct URL to the resource, but that would seriously compromise anonymity.

\section{COMPARING Q/A TO GNUTELLA}

The primary operational distinction between Gnutella and $\mathrm{Q} / \mathrm{A}$ is the use of explicit advertisements. Gnutella file providers give no previews about their available files. This forces all query messages to be delivered to all providers, and the equivalent of filtering is only performed at the last step by the provider.

The management of anonymity in Q/A differs from Gnutella in an important way. As indicated in Section 2, Gnutella's anonymity is limited since messages must sometimes use explicit IP addresses, causing them to be visible to all clients. In Q/A, clients only need to provide their IP address to the first level server to which they connect. After that, all references to the client are in terms of content-based advertisements and addresses. This effectively hides the identity of clients from all other servers and clients comprising the network.

The primary architectural distinction between Gnutella and Q/A is the separation of clients and servers. Q/A assumes a set of distinguished nodes that are running a specific server software package. The servers are interconnected and use a protocol that is different from the one used between client and server. Q/A clients use some out-of-band mechanism to locate a server. The client connects to that server and generates messages and provides advertisements for receiving messages. It is important to note that Q/A clients do not participate in message routing. That functionality is reserved to the servers. The architecture is further distinguished by its ability to selectively route messages based on 
forwarding of advertisements and on optimizations provided by the Covers relations among advertisements.

We can now revisit our list of Gnutella flaws and see how Q/A solves many of these problems.

- Anonymity. Q/A's anonymity is more complete than is Gnutella's because only a single server needs to know the IP address of a client.

- Efficiency. The use of advertisements and the Covers relation significantly reduces the traffic in a Q/A net as opposed to a Gnutella net. Q/A also automatically routes replies along the path from the advertiser to the query originator based on the originators reply address.

- Malicious Users. If the set of servers is controlled carefully, the impact of a malicious client can be limited because it can only affect the first level client-server communication. Malformed messages from a client can be caught and suppressed by its server. Also, any attempt by a client to flood the network with queries can be metered by a server, and queries for which there is no possible provider will be suppressed and not propagated since no filter will match.

- $\quad$ Query Expressions. Q/A query expressions are standardized by the underlying Publish/Subscribe system so there is never any misunderstanding about their interpretation. There is, however an issue about their general expressiveness (see Section 7.2).

The Q/A approach has many advantages and fixes many of the problems of Gnutella-like systems, In fairness, however, there are some advantages for the Gnutella approach that a Q/A system would currently find difficult to emulate.

One advantage is the ability of clients also to act as servers and to dynamically extend the Gnutella network. Q/A inherently requires a separation of servers and clients, and it is this separation that provides many of the Q/A advantages. Still, this is a very desirable property and worthy of further study.

A second advantage concerns caching. Some variants of the Gnutella architecture support caching of files at more than one client. This is possible because of the spreading activation model, because clients are part of the routing infrastructure, and because many queries are requests for specific files. Adding caching to the Q/A architecture is potentially possible, but would appear to require major changes in the current message processing, and would require the embedding of knowledge about resources into the Q/A servers. In effect, a mobile resource would need to carry its advertisement along with it.

\section{RESEARCH ISSUES}

Although this paper presents a reasonable design and implementation for Q/A using a specific Publish/Subscribe system, there is clearly much room for additional research and for alternative designs with other desirable properties. This section identifies some of those research issues and discusses some possible alternatives.

\subsection{Response Collection}

Replies to queries are returned asynchronously with respect to the original query. So an important problem is determining when all responses to a query have been received. Depending on client speed, client response size, server routing, and network size, responses can appear arbitrarily long after the original query.

We can identify a number of standard solutions that help address this problem of collecting responses.

- Timeout - wait for a specified period of time and take whatever responses have been received by that time.

- Number-of-Responses - wait until a specified number of responses have been returned. This assumes that the network is reliable and that all - or enough - clients are up to provide the required number of responses.

- Two-Phase-Query - wait for a period of time and then send out a follow-up query that forces responders to generate an immediate response.

- Quick-Reply - upon receiving a message, advertisers generate an immediate reply saying, in effect, "I will reply."

- Extended-Query - accept arbitrarily late responses. This presumes that it makes sense to process query responses individually.

In many cases, some solutions can profit from or require the use of other solutions. Two-phase-query, for example, requires the use of timeout, and number-of-responses could profit from quickreply information.

\subsection{Expressiveness}

Using simple attribute-value pairs for queries may not be as expressive as required for realistic queries. Instead, one might prefer to have queries that can specify such things as regular expressions for strings or value ranges for numbers. On the advertisement side, tuples with comparison operators are already supported, but again expressiveness may be lacking. The problem with moving to more expressive queries and advertisements is that it can significantly complicate routing by the Q/A servers because it makes computing the Covers relation difficult and reduces opportunities for optimization.

In the event that an advertiser has multiple matching resources, it must return multiple responses to the query originator. The simplest approach is to generate multiple responses: one for each matching resource. This is the approach taken in the prototype (Section 5). The problem with this approach is that it leads to significantly more message traffic. An alternative is to encode all of the responses from a given advertiser into a single reply message. Given the simple attribute-value pair format of our prototype, this encoding is non-trivial. Solving this requires adding some form of list or vector type to the current format.

\subsection{Resource Retrieval}

Even after a client has received responses to its query, it still has the task of retrieving that resource from some responding client. Conceptually, the simplest solution is for the provider of the resource to send its contents back through the Q/A network to the consumer of that resource. This assumes that such transmission makes sense for the resource. Using the Q/A network maintains complete anonymity and uses the same infrastructure for all communications, but at the cost of increased message traffic.

In Gnutella, the retrieval process occurs out-of-band. That is, the query node connects directly to the response node and uses a simplified version of the HTTP protocol to retrieve the file. Q/A could use such an approach, but naively implemented, it 
significantly reduces client anonymity because one or the other of the clients must reveal its IP address so the connection can be made. One possible solution is to use a well-known (and trusted) third party (e.g., one of the Q/A servers). Both clients connect to the third party, which then provides a channel for passing information from one client to the other. This has the advantage of keeping the initial clients anonymous with respect to everyone except the third party.

\subsection{Malicious Activities}

Although Q/A can prevent many malicious activities, it shares with other Gnutella-like systems some vulnerabilities that are harder to stop.

- False Advertising. Consider, for example, a client that advertises for the equivalent of "all messages." When it receives a message, it responds with a bogus reply containing an advertisement for a pornographic site. A Q/A server could mitigate this by requiring all advertisements to have a degree of specificity. For popular resources, this might not provide much protection.

- Freeloading. It has been found empirically [1] that most users of Gnutella retrieve resources, but do not themselves ever provide any resources to others. A market-based approach, such as used by MojoNation [16][18], might alleviate this problem, but adding this kind of capability to a Q/A system or to the routing infrastructure of a Publish/Subscribe system remains an open research issue.

\subsection{Performance}

The performance of the simple Siena-based prototype is adequate for small-scale use. Some Siena simulations [5] exist that provide plausible evidence that the underlying content-based routing network is scalable. Similar simulations combined with performance measurements are needed to verify the scalability of a Q/A system.

\subsection{Long-Term Queries}

The Q/A model as described in this paper requires a query client to periodically send out a query in order to detect new replies that may have become available since the last query. It would be more desirable if this could be automated in some fashion so that new replies could be sent as soon as they become available.

One way to achieve this is to combine Q/A directly with the Publish/Subscribe paradigm. Thus, some queries could be marked as, say, persistent, so that they would remain in force and would continue to receive replies until rescinded. Operationally, this would be implemented by turning the query into a subscription, and introducing a mechanism by which advertising clients could inform their interface of new resources. If the new resource matched a persistent query, then an event message (in the Publish/Subscribe sense) would automatically be generated and sent to the query client.

\section{RELATED WORK}

The closest systems related to Gnutella and Q/A are Napster [19][25] and Freenet [7]. Unfortunately, little information about these systems exists outside of the World Wide Web. So for those web-based references, the bibliographic citations in Section 11 include a specific URL.
The primary difference between Napster and Gnutella is in the architecture. Napster uses a restricted set of centralized servers to do the query and advertise matching. It is similar to Gnutella in that the files are held in user established clients and the set of clients can grow and shrink dynamically.

Freenet provides anonymous distributed file sharing, and it is extensible by user-established clients. It has a much more restricted notion of query than does $\mathrm{Q} / \mathrm{A}$ : clients ask for specific files (identified by a unique hash) and the search process stops when that specific file is found. Caching is also supported. Freenet uses message traffic about as efficiently as does Siena's content-based routing, and far more efficiently than Gnutella. It is not clear how Freenet could be extended to support more general resource discovery because the only property supported by Freenet is a hash of the file contents. All querying and caching is based on this hash, and that hash has no semantic meaning. Thus no form of relational query is possible.

MojoNation [16][18] is a more recent system that attempts to reduce message traffic and freeloading by adding a charging model. Downloading a file incurs "costs" and providing a file for others provides "money" to use against costs. It is possible to add such a capability to Quad since it is orthogonal to other issues that MojoNation does not address: anonymity, scale, and query.

The Quad Q/A system is built upon Siena, but other Publish/Subscribe systems are available as alternatives upon which to build a Q/A system. There are two issues here: scalability to wide-area networks and expressiveness. Most Publish/Subscribe systems are designed for local-area network use. Examples are Field [21] and ToolTalk [14]. Two systems other than Siena address the wide-area network issue: TIBCO [23] and Elvin [22]. The primary problem with both is their lack of automatic Covers relation support. The equivalent of Covers relations must be manually established and maintained.

Expressiveness is a problem for subject-based (also known as topic-based) Publish/Subscribe systems. These systems, of which TIBCO is an example, provide only a single content string (the subject) for use in routing. This severely limits expressiveness, and it is not clear if any sort of reasonable Q/A system could be built using a subject-based system.

Resource discovery systems are tangentially related to Q/A. Jini $^{\mathrm{TM}}[13][24]$ is perhaps the best known of these systems. Jini defines a collection of programming interfaces. The implementations behind them are prototypes that do not appear to have addressed issues such as wide-area scale and message traffic. In principle, there is no reason that the resource discovery part of Jini could not be realized by a Q/A system.

\section{CONCLUSION}

This paper demonstrates that Publish/Subscribe middleware can be used to construct a Query/Advertise system with functionality essentially equivalent to Gnutella. A prototype Q/A system was implemented over the Siena Publish/Subscribe system to demonstrate the feasibility of this approach.

Further, using a Publish/Subscribe system solves or mitigates many of the known problems of Gnutella. On the negative side, a number of research problems have been identified whose solution would improve the ability of a Publish/Subscribe messaging system to support the Query/Advertise paradigm. 


\section{ACKNOWLEDGEMENTS}

Alexander Wolf, Richard Hall, and Antonio Carzaniga provided valuable comments about the relationship of Gnutella and Publish/Subscribe. Antonio Carzaniga also provided Siena.

This material is based upon work sponsored by the Air Force Materiel Command, Rome Laboratory, SPAWAR, and the Advanced Research Projects Agency under Contract Numbers F30602-00-2-0608 and N66001-00-8945. The content of the information does not necessarily reflect the position or the policy of the Government and no official endorsement should be inferred.

\section{REFERENCES}

[1] E. Adar and B. A. Huberman. Free Riding on Gnutella. Technical report, Xerox PARC, 10 Aug. 2000.

[2] A. Carzaniga. Siena: A Wide-Area Event Notification Service. University of Colorado Software Engineering Research Laboratory (SERL). http://www.cs.colorado.edu/serl/dot/siena.html.

[3] A. Carzaniga, D. Rosenblum, and A. Wolf. Achieving Expressiveness and Scalability in an Internet-Scale Event Notification Service. In Proc. of the 19th ACM Symposium on Principles of Distributed Computing, Portland OR., July 2000.

[4] A. Carzaniga, D. R. Rosenblum, and A. L. Wolf. Challenges for Distributed Event Services: Scalability vs. Expressiveness. In Engineering Distributed Objects '99, Los Angeles CA, USA, May 1999.

[5] A. Carzaniga, D. S. Rosenblum, and A. L. Wolf. Interfaces and Algorithms for a Wide-area Event Notification Service. Technical Report CU-CS-888-99, Department of Computer Science, University of Colorado, Oct. 1999. Revised May 2000.

[6] A. Carzaniga, D. S. Rosenblum, and A. L. Wolf. Content-based Addressing and Routing: A General Model and its Application. Technical Report CU-CS902-00, Department of Computer Science, University of Colorado, Jan. 2000.

[7] I. Clarke, O. Sandberg, B. Wiley, and T. W. Hong. Freenet: A Distributed Anonymous Information Storage and Retrieval System. In Proc. of the ICSI Workshop on Design Issues in Anonymity and Unobservability, Berkeley, CA, 2000. International Computer Science Institute.

[8] R. Fielding, J. Gettys, J. Mogul, H. Frystyk, L. Masinter, P. Leach, and T. Berners-Lee. Hypertext transfer protocol - http/1.1. Technical Report RFC2616, IETF, 1999.
[9] Gnutella Home Web Page. http://gnutella.wego.com/.

[10] Gnutella Developers Home Web Page. http://gnutelladev.wego.com.

[11] Knowbuddy's Gnutella FAQ. http://www.lysator.liu.se/ $\sim$ mitja/protest/gnutellafaq.html.

[12] D. Heimbigner. Adapting Publish/Subscribe Middleware to Achieve Gnutella-like Functionality. Technical Report CU-CS-909-00, Department of Computer Science, University of Colorado, Sept. 2000. http://www.cs.colorado.edu/serl/dot/Papers.html.

[13] Jini ${ }^{\text {TM }}$ Specification, version 1.1 Beta, 1999.

[14] A. M. Julienne and B. Holtz. ToolTalk and Open Protocols, Inter-Application Communication. PrenticeHall, 1994.

[15]L. Kahney. Intel Says: Think like Napster. Wired News, 2000. http://www.wired.com/news/technology/ 0,1282,38413,00.html.

[16]D. McCullagh. Get Your Music Mojo Working. Wired News, 2000. http://www.wired.com/news/technology/ 0,1282,37892,00.html.

[17] P. McDougall. The Power of Peer-To-Peer. Information Week, 2000. http://informationweek.com/801/peer.htm.

[18] Mojo Nation Home Web Page. http://www.mojonation.net/.

[19] Napster Home Web Page. http://www.napster.com/.

[20] The Working Group on Peer-To-Peer Computing. http://www.peer-to-peerwg.org.

[21] S. P. Reiss. Connecting Tools Using Message Passing in the Field Environment. IEEE Software, pages 57-67, July 1990.

[22] W. Segall and D. Arnold. Elvin Has Left the Building: A Publish/Subscribe Notification Service with Quenching. In Proc. of the 1997 Australian UNIX Users Group, Brisbane, Australia, Sept. 1997.

[23] TIBCO, Inc. Rendezvous Information Bus, 1996. http://www.rv.tibco.com/rvwhitepaper.html.

[24] J. Waldo. Jini ${ }^{\mathrm{TM}}$ Architectural Overview: Technical White Paper. Technical report, Sun Microsystems, 1999.

[25] J. Zien. The Technology Behind Napster. About, 2000. http://internet.about.com/library/weekly/2000/ aa052800b.htm. 